\title{
On Discrete Cosmology, Gravitation and Mechanics
}

\author{
José Alcauza \\ Department of Technology, Telefónica Movistar, Madrid, Spain \\ Email: jose.alcauzaatencia@telefonica.com
}

How to cite this paper: Alcauza, J. (2019) On Discrete Cosmology, Gravitation and Mechanics. Journal of High Energy Physics, Gravitation and Cosmology, 5, 1150-1182. https://doi.org/10.4236/jhepgc.2019.54066

Received: August 26, 2019

Accepted: October 19, 2019

Published: October 22, 2019

Copyright $\odot 2019$ by author(s) and Scientific Research Publishing Inc. This work is licensed under the Creative Commons Attribution International License (CC BY 4.0).

http://creativecommons.org/licenses/by/4.0/

\begin{abstract}
In this paper we will discretely reformulate the main fundamental magnitudes of mechanics and thermodynamics due to a new dynamic, discrete and irreversible nature for Time. The existence of a fundamental minimum time, implies that any physical system can only evolve discreetly according to this minimum time instead of a continuous evolution. Thus, the passage of Time must be considered a fundamental physical process and incorporated into Physics where the laws of Nature depend on a clear distinction between past, present and future. A time interval equals a loss of energy. The introduction of "dark matter", "dark energy", "ad hoc modifications of the laws of mechanics" or "fundamental constants varying" will prove to be unnecessary inasmuch as the view here to be developed will not require of a Universe provided with special properties. By considering that Universe can be expressed as the ensemble of $N$ typical particles in motion of mass $m$, we will find possible solutions to some of the main problems of the current Physics, all from an existing deep connection between gravity, thermodynamics and quantum cosmology.
\end{abstract}

\section{Keywords}

Discrete Time and Space, Discrete Cosmology, Passage of Time, Discrete Entropy

\section{Introduction}

One of the main problems in modern physics is the temporal irreversibility, that is, the difficulty of its fundamental theories in distinguishing between the past and the future. How is it possible that temporal irreversibility of macroscopic processes emerges from fundamental physical laws of reversible character?

As is well known, in the present physical laws of Newtonian Mechanics and 
Relativity, a mysterious temporal symmetry is established, that is, the directional character of Time is not distinguished. Quantum Mechanics also maintains this reversible character, although the role of the observer through the measurement process, makes it become irreversible through the "collapse" of the wave function. Thermodynamics through the second law is the one that most clearly indicates the need for a direction of Time: The loss of a part of unrecoverable energy in the form of heat, makes the physical processes irreversible and gives the Time a unique direction allowed for the future.

Also there exist in Nature the known examples of temporal irreversibility or so-called time's arrows ${ }^{1}$ which clearly indicate a unique direction towards the future. In addition, recently in [1], the authors have revealed the observation of the non-Abelian Aharonov-Bohm effect that violates temporal symmetry and gives time a unidirectional character.

All of it rather suggests that passage of Time must be incorporated into Physics as a fundamental process by which Time is always advancing in a unique direction: in positive direction towards the future. We will raise this conjecture, the purport of which will hereafter be called the "Passage of Time Principle", to the status of a postulate. This postulate is sufficient to find possible solutions to some of the main problems of the current physics, because the difficulties being encountered today in the explanation of several cosmological and quantum phenomena, lie at the root of an insufficient consideration on concept of passage of Time and its implications in physical systems.

\section{Kinematical Part}

The following reflections are based on the principle previously mentioned and we define it as follows:

Passage of Time Principle: The passage of Time is a fundamental physical process by which Time always is advancing in a unique direction: in positive direction towards the future.

This principle is valid for any reference system in Nature. Therefore we can conclude that:

- Causality comes directly from the Passage of Time Principle.

- Any Time value determined with regard to any relative reference system is always positive and therefore no process associated with temporal reversibility can exist in the Universe: there are no causal loops or regions or phenomena where Time "evolves backward".

However, we know that all physical process is defined as the set of successive phases or states of a system, that is, the set of one or more state changes until a concrete state. We can never define a physical process at a given time because any physical process needs a time interval to be completed. The simpler the

${ }^{1}$ The thermodynamic arrow of time that is provided by the second law of Thermodynamics, the cosmological arrow of time that points in the direction of the universe's expansion, the causal arrow of time: all cause precedes its effect, the quantum arrow of time due to wave function collapse, the psychological/perceptual arrow of time, etc. 
process, the smaller the time interval. Thus, if a physical process consists of $n$ successive subprocesses, every subprocess will need a minimum interval of time to be completed and therefore the total time interval of the physical process will be the sum of the minimum intervals of time of every subprocess.

According to this principle, the passage of Time is a fundamental physical process, therefore must exist a minimum interval of Time in Nature that will be the simplest physical process and which we will call "fundamental minimum time". Thus, the passage of Time will be done discreetly by successive advances of this fundamental minimum time and not continuously.

Let us denote this fundamental minimum time as $\tau$. Therefore, any relative interval of time will consist in a sequence of this fundamental minimum time, that is, all time value $\Delta t$ determined with regard to any reference system (proper time interval), can be expressed by the equation:

$$
\Delta t=t_{n} \quad \Leftrightarrow \quad t_{n}=\sum_{1}^{n} \tau=n \cdot \tau
$$

With $n \in \mathbb{Z}^{+}$, that is: $n=1,2,3, \cdots$ the number of intervals until $t_{n}$.

By definition, this fundamental minimum time $\tau$ is constant for all relative reference system in Nature. According to Relativity, if for a given relative reference system $K$ we have: $\Delta t=n \cdot \tau$; then for a system $K^{\prime}$ that is in any state of motion with regard to $K$ we will have: $\Delta t^{\prime}=n^{\prime} \cdot \tau$, that is, what varies with regard to every reference system is only the number of intervals. Faizal et al., in [2] described a discrete spectrum for time of several orders of magnitude greater than the Planck scale which are consistent with fundamental minimum time.

\subsection{Universe's Time and Fundamental Minimum Time}

The age, cosmological Time or Time of the Universe $t$ with regard to any relative reference system, is the elapsed time from the origin of the Universe ${ }^{2}$ to any Time $t$. Therefore according Passage of Time Principle, Universe's Time $t$ can be expressed with regard to any relative reference system, by the equation:

$$
t=\sum_{1}^{n_{t}} \tau=n_{t} \cdot \tau
$$

With $n_{t} \in \mathbb{Z}^{+}$, that is: $n_{t}=1,2,3, \cdots$ the number of intervals until $t$. In what follows, when we define the value of any physical quantity at Time $t$, we are referring to this Time of the Universe $t$.

We know Planck time is defined as:

$$
t_{P}=\sqrt{\frac{\hbar G}{c^{5}}}=\frac{\hbar}{m_{P} c^{2}} \Leftrightarrow m_{P}=\sqrt{\frac{\hbar c}{G}}
$$

where $\hbar=\frac{h}{2 \pi}$ is the reduced Planck constant, $G$ is the constant gravitation, $c$ is the constant of the speed of light in the vacuum and $m_{P}$ is the so-called Planck ${ }^{2}$ According to the current theory of the Big Bang, which may be considered until now as the most prudent and widely accepted model of the formation of the Universe. 
mass.

However, by definition, the fundamental minimum time $\tau$ is constant for all relative reference system in Nature, thus, since the Planck time $t_{P}$ is also always constant for all relative reference system because it is a function of the three fundamental constants, we can define $\tau$ as the product of a positive real constant $k$ by Planck time, that is:

$$
\tau=k \cdot t_{P}=k \cdot \frac{\hbar}{m_{P} c^{2}} \Leftrightarrow\left(k \in \mathbb{R}^{+} ; \forall k>0\right)
$$

Thus, we can now express Equation (2) as:

$$
t=\left(n_{t} \cdot k\right) \frac{\hbar}{m_{P} c^{2}}
$$

If we now multiply both terms of this equation by $\frac{\hbar}{G}$, equation does not change, and then according to Planck mass $m_{P}$, we obtain:

$$
m_{P} \cdot \frac{\hbar c}{G}=\left(n_{t} \cdot k\right) \frac{\hbar^{2}}{c G t} \Rightarrow m_{P}^{3}=\left(n_{t} \cdot k\right) \frac{\hbar^{2}}{c G t}
$$

Now, let us establish that with regard to any relative reference system, the total Mass of the Universe $M$ at Universe's Time $t$ can be expressed as the ensemble of $N$ typical particles in motion of mass $m$. Thus:

$$
M=N \cdot m
$$

where $N$ y $m$ also are defined at Time $t$ and $\left(N \in \mathbb{Z}^{+}\right)$is a positive integer with $N \geq 1$.

Therefore, since that any typical material particle of mass $m$ in motion can be expressed at any Time $t$ as a function of Planck mass $m_{P}$ as:

$$
m=\beta \cdot m_{P} \Leftrightarrow\left(\beta \in \mathbb{R}^{+} ; \beta \neq 0\right)
$$

We can replace this value in Equation (3) and we obtain for any reference system the equation:

$$
m^{3}=\Omega \cdot \frac{\hbar^{2}}{c G t} \Leftrightarrow t=\Omega \cdot \frac{\hbar^{2}}{c G m^{3}}
$$

which is the Weinberg's relation [3] for any typical material particle of mass $m$ in motion at Time $t$ and where obviously: $\left[\Omega=n_{t} \cdot k \cdot \beta^{3} \Leftrightarrow\left(\Omega \in \mathbb{R}^{+} ; \Omega \neq 0\right)\right]$.

However, Equation (5) can be now expressed according Equation (4) as:

$$
\sqrt{\frac{G M}{c^{3}} t}=\sqrt{N} \cdot \sqrt{\Omega} \cdot \frac{\hbar}{m c^{2}}
$$

Then by simple equating this equation with Equation (2) for Universe's Time, that is: $t=n_{t} \cdot \tau$, we obtain for any relative reference system:

1) Universe's Mass as a function of Universe's Time

$$
t=\sqrt{\frac{G M}{c^{3}} t} \Rightarrow M=\frac{c^{3}}{G} t
$$

This equation, in addition to determine the Mass of the Universe at Time $t$, 
implies by the Passage of Time Principle that Universe's Mass must increase as Time progresses.

2) Fundamental Minimum Time

$$
\tau=\sqrt{\Omega} \cdot \frac{\hbar}{m c^{2}} \Rightarrow \tau=\sqrt{\frac{G m t}{c^{3}}}
$$

where we have replaced the value $\Omega$ of Equation (5).

3) Universe Time

$$
n_{t}=\sqrt{N} \Rightarrow t=\sqrt{N} \cdot \tau
$$

The number of intervals to determine the Time of the Universe $t$ according to Equation (2), is the square root of the total number $N$ of typical particles in motion of mass $m$ in the Universe at Time $t$.

4) Universe Particles Number

$$
N=\frac{c^{3} t}{G m}
$$

By Equation (4) and (6) we can express the total number $N$ of typical particles in motion of mass $m$ in the Universe at Time $t$.

\section{Proper Time}

According previous section, the Time of the Universe $t$ can be expressed as:

$$
t=\sqrt{N} \cdot \tau
$$

where $N$ is the total number $N$ of typical particles in motion of mass $m$ in the Universe at Time $t$ according Equation (8) and $\tau$ id the fundamental minimum time according Equation (7).

Let us now consider with regard to any relative reference system, an initial value of Universe Time $t_{i}$ and any later value $t$, with $t>t_{i}$, where therefore an interval of time has elapsed $\Delta t=t-t_{i}$. Thus, according to Equation (1) and Equation (9), all time interval $\Delta t$ with regard to any relative reference system, that is its proper time interval, can be expressed as the interval of Universe's Time elapsed with regard to that reference system:

$$
\Delta t=t-t_{i}=n \cdot \tau \Rightarrow \Delta t=n \cdot \sqrt{\frac{G m t}{c^{3}}} \Leftrightarrow n=\sqrt{N(t)}-\sqrt{N\left(t_{i}\right)}
$$

where $N(t)$ and $N\left(t_{i}\right)$ are the total number of typical particles in motion of mass $m$ and $m_{i}{ }^{3}$ at Universe's Time $t$ and $t_{i}$ respectively according to Equation (8).

Obviously according to Relativity, if for any relative reference system $K$ we have for the Time of the Universe $t=\sqrt{N} \cdot \tau$ and for any proper time interval $\Delta t=t-t_{i}=n \cdot \tau$ with $n=\sqrt{N(t)}-\sqrt{N\left(t_{i}\right)}$, then for any system $K^{\prime}$ in any state of motion with regard to $K$ we will have: $t^{\prime}=\sqrt{N^{\prime}} \cdot \tau$ for Universe Time and $\Delta t^{\prime}=t^{\prime}-t_{i}^{\prime}=n^{\prime} \cdot \tau$ for the proper time interval with $n^{\prime}=\sqrt{N^{\prime}\left(t^{\prime}\right)}-\sqrt{N^{\prime}\left(t_{i}^{\prime}\right)}$. ${ }^{3}$ In Section $\$ 3.1$ we will prove that all material particle must lose an unrecoverable quantity of mass/energy as Time progresses. 


\subsection{Proper Interval of Space. Fundamental Minimum Length}

By Equation (7) for the fundamental minimum time $\tau$, we verify that the product:

$$
l=c \cdot \tau \Rightarrow l=\sqrt{\frac{G m t}{c}}
$$

Is a fundamental minimum length which we denote by 1 . Since $\tau$ is constant for any relative reference system and $c$ is a fundamental constant, then $l$ is equally constant for any relative reference system. Therefore, we can express any length $\Delta r=r-r_{i}$ (proper interval of space) with regard to any relative reference system, as:

$$
\Delta r=\sum_{1}^{n_{r}} l=n_{r} \cdot l \Rightarrow \Delta r=n_{r} \cdot \sqrt{\frac{G m t}{c}} \Rightarrow r=r_{i}+n_{r} \cdot \sqrt{\frac{G m t}{c}}
$$

where $r$ and $r_{i}$ are the particle's position at Time $t$ and $t_{i}$ respectively and $\left(n_{r} \in \mathbb{Z}\right)$, as number of steps to $r_{n}$. Obviously this length will be positive + or negative - depending on the spatial direction with regard to the origin of the relative reference system that is defined.

Also according to Relativity, if for any relative reference system $K$ we have a relative proper interval of space $r_{n}=n_{r} \cdot l$, then for any system $K^{\prime}$ in any state of motion with regard to $K$ we will have: $r_{n^{\prime}}^{\prime}=n_{r}^{\prime} \cdot l$.

\subsection{Discrete Velocity of a Material Particle. Minimum Velocity}

Let us now consider any relative reference system. We establish that with regard to this reference system, the velocity $v$ of any material particle at Time $t$ can be expressed of simple form according to Equation (12) and Equation (10) respectively, as:

$$
v=\frac{\Delta r}{\Delta t} \Rightarrow v=\left(\frac{n_{r}}{n}\right) c
$$

where obviously $\left(n_{r} \leq n \Leftrightarrow v \leq c\right)$ for Relativity. Therefore the equation of motion will be:

$$
r=r_{i}+v \Delta t
$$

where $r_{i}$ is the particle's initial position at Time $t_{i}$. However, by Equation (57) we can express de speed of de light $c$ at Time $t$ as:

$$
c=\sqrt{N} \cdot \frac{l}{t} \Rightarrow c=\sqrt{N} \cdot v_{\min } \Leftrightarrow v_{\min }=\frac{l}{t}=\sqrt{\frac{G m}{c t}}
$$

where $l$ is the fundamental minimum length according to Equation (11) and where we can establish $v_{\min }$ as minimum velocity of any material particle at Time $t$. It's remarkable that $v_{\min }$ is not constant as Time progresses. Therefore, any material particle only can have the discrete velocity $v$ at Time $t$ as:

$$
v=n_{v} \cdot v_{\min }=n_{v} \cdot \frac{l}{t} \Rightarrow v=n_{v} \cdot \sqrt{\frac{G m}{c t}}
$$


where $\left(n_{v} \in \mathbb{Z}^{+}\right)$is a positive integer. If we now express $\left(v_{i}=n_{v_{i}} \cdot \frac{l}{t_{i}}\right)$ as the particle's velocity at Time $t_{i}$ with $\left(n_{v_{i}} \in \mathbb{Z}^{+}\right)$, then any velocity variation $\Delta v=v-v_{i}$ during Time elapsed $\Delta t=t-t_{i}$ can be expressed as:

$$
\Delta v=n_{\alpha} \cdot v_{\min } \Leftrightarrow \Delta v=n_{\alpha} \cdot \sqrt{\frac{G m}{c t}}
$$

where $\left[n_{\alpha} \in \mathbb{Z} ; n_{\alpha}=n_{v}-n_{v_{i}}\left(\frac{t}{t_{i}}\right)\right]$ is a integer and will be positive + or negative - depending if velocity variation increases or decreases. Obviously if, at Time $t_{i}$, for a relative reference system the material particle is at rest $\left(v_{i}=0\right)$, then $\left(n_{v_{i}}=0\right)$ and therefore $\left(n_{\alpha}=n_{v}\right)$ and the velocity $v$ at Time $t$ will be according to Equation (16).

\subsection{Fractional Acceleration of a Material Particle}

Let us now consider any relative reference system. We establish that with regard to this reference system the acceleration a of any material particle at Time $t$ can be expressed according to Equation (10) and Equation (17) as:

$$
a=\frac{\Delta v}{\Delta t} \Rightarrow a=\left(\frac{n_{\alpha}}{n}\right) \cdot \frac{c}{t}
$$

where $\left(n \in \mathbb{Z}^{+}\right)$and $\left(n_{\alpha} \in \mathbb{Z}\right)$ is a integer and will be positive + or negative depending on velocity variation if increases or decreases according to Equation (17).

\section{Equation of Motion}

By Equation (18) any variation of velocity $\Delta v=v-v_{i}$ during Time elapsed $\Delta t=t-t_{i}$ with regard to any relative reference system can be expressed as:

$$
v=v_{i}+a \Delta t \Rightarrow r=r_{i}+v_{i} \Delta t+a(\Delta t)^{2}
$$

According to Equation (14) and where $r_{i}, v_{i}$ are the particle's initial position and velocity at Time $t_{i}$ respectively.

\subsection{Velocity-Distance Law. Fractional $H(t)$ Parameter}

Let us now consider any relative reference system. According Equation (12) for any length $\Delta r$ (proper interval of space), we can now express Equation (16) as:

$$
v=n_{v} \cdot \sqrt{\frac{G m}{c t}} \Rightarrow v=\left(\frac{n_{v}}{n_{r}}\right) \frac{1}{t} \cdot n_{r} \sqrt{\frac{G m t}{c}}
$$

And therefore:

$$
v=H(t) \cdot \Delta r \Leftrightarrow H(t)=\left(\frac{n_{v}}{n_{r}}\right) \frac{1}{t}
$$

where $\left(n_{v}, n_{r}\right) \in \mathbb{Z}^{+}$obviously are a positive integers. Thus, we obtain the Velocity-Distance Law and fractional $H(t)$ parameter as a function of Universe's 
Time and as a consequence of discrete velocities of the material particles. It is remarkable that this equation is not only applicable to great distances but has a general character at any distance.

\section{Fractional Acceleration as a Function of $H(t)$}

Let us now consider any relative reference system. We establish that at Time $t_{i}$, the particle's initial velocity is: $v_{i}=0$. Therefore, according to Equation (19) for the velocity: $v=v_{i}+a \Delta t$ and Equation (20), the velocity and acceleration of material particle at Time $t$ can be expressed as:

$$
v=a \Delta t=H(t) \cdot \Delta r \Rightarrow H(t)=\frac{a}{v}
$$

where obviously $v=\frac{\Delta r}{\Delta t}$. Therefore, by Equation (13), we can also express the fractional acceleration of any material particle at any Time $t$ as:

$$
a=v \cdot H(t) \Rightarrow a=\left(\frac{n_{r}}{n}\right) \cdot c H(t)
$$

where obviously $\left(n \in \mathbb{Z}^{+}\right)$and $\left(n_{r} \in \mathbb{Z}\right)$.

\section{Dynamical Part}

\subsection{On passage of Time as Irreversible Physical Process}

Let us consider any relative reference system. According Equation (7) for the fundamental minimum time we obtain:

$$
\tau=\sqrt{\frac{G m t}{c^{3}}} \Rightarrow m \cdot t=\frac{c^{3}}{G} \tau^{2}
$$

We also establish that this reference system is isolated, that is, on the material particle does not act any force and that the material particle is at rest. According Passage of Time Principle and as $m \cdot t \equiv c t e$ by the constancy of the fundamental minimum time $\tau$ for any reference system, the mass $m$ of material particle must decrease as Time progresses. Thus is, if we consider any interval of proper time $\Delta t=t-t_{i}$ according Equation (10), the variation of Equation (23) with regard to this proper time interval $\Delta t$ will be:

$$
\frac{\Delta}{\Delta t}(m \cdot t)=\frac{\Delta}{\Delta t}\left(\frac{c^{3}}{G} \tau^{2}\right)=0 \Rightarrow \frac{\Delta m}{\Delta t} \cdot t+m=0
$$

And therefore finally we obtain:

$$
\frac{\Delta m}{\Delta t}=-\frac{m}{t} \Rightarrow \frac{\Delta E}{\Delta t}=-\frac{m c^{2}}{t}
$$

where obviously $E=m c^{2}$ is the energy of the particle at Universe's Time $t$ by Equation (26).

Therefore, according to Passage of Time Principle we can conclude that any material particle of the Universe must lose an unrecoverable quantity of mass/energy as Time progresses according Equation (24) converting the passage 
of Time in a irreversible physical process, the irreversibility in a fundamental property in Nature and establishing a clear distinction between past, present and future. Thus, all the physical magnitudes of any system in Nature must be specified with regard to Universe's Time $t$. One way to verify the Passage of Time is to determine this lost mass/energy.

\section{A Time Interval Equals a Loss of Energy}

It's remarkable that from Equation (24) we can obtain the following relationships:

$$
\frac{\Delta E}{\Delta t}=-\frac{E}{t} \Rightarrow\left\{\begin{array}{l}
\Delta E=-\eta \cdot \Delta t \\
E=\eta \cdot t
\end{array}\right.
$$

where $\eta \in \mathbb{R}^{+}$is a constant. Therefore any time interval equals a loss of energy: $\Delta E=-\eta \cdot \Delta t$.

\subsection{Energy and Frequency of a Material Particle}

Consider any relative reference system. By selecting the first equation in Equation (7) for the fundamental minimum time, Equation (5) for $\Omega, \hbar=\frac{h}{2 \pi}$ and Equation (9) for the Time of the Universe, we can then express the energy $E=m c^{2}$ of any material particle at Time $t$ as:

$$
m c^{2}=\hbar \cdot \frac{\sqrt{\Omega}}{\tau} \Rightarrow m c^{2}=h v \Leftrightarrow v=\sqrt{\frac{N G m^{3} c}{h^{2} t}}
$$

with $v$ as a fundamental oscillation frequency associated with every particle $m$ at Time $t$. Thus, we will have for any material particle of the universe in any state of motion, the relation: $E=h \cdot v$.

However, we know by wave mechanics the relationship between the frequency of a wave $v$, its wavelength $\lambda$ and the propagation velocity or phase velocity $v_{p}$, that is:

$$
\lambda \cdot v=v_{p}
$$

Therefore, according to Equation (26) we can write this previous equation as:

$$
\lambda \cdot \sqrt{\frac{N G m^{3} c}{h^{2} t}}=v_{p}
$$

According Equation (8), we obtain for the wavelength associated to the particle at Time $t$.

$$
\lambda=\frac{h v_{p}}{m c^{2}} \Rightarrow \lambda=\frac{h}{m v} \Leftrightarrow v_{p} \cdot v=c^{2}
$$

That is the famous De Broglie wavelength and where $v=\frac{c^{2}}{v_{p}}$ is the velocity of the material particle $m$ at Universe Time $t$.

\subsubsection{Minimum Energy and Mass}

According to Equation (26) we can also express the energy $E$ of any material 
particle at Time $t$ as:

$$
E=m c^{2}=\sqrt{N} \cdot \sqrt{\frac{G m^{3} c}{t}} \Rightarrow E=\sqrt{N} \cdot E_{\min } \Leftrightarrow E_{\min }=\sqrt{\frac{G m^{3} c}{t}}
$$

where $E_{\min }$ is the minimum energy at Time $t$. Therefore, now we can express the minimum mass in nature $m_{\min }$ or "bit" (the minimum information) at Time $t$ as:

$$
m=\sqrt{N} \cdot \sqrt{\frac{G m^{3}}{c^{3} t}} \Rightarrow m=\sqrt{N} \cdot m_{\min } \Leftrightarrow m_{\min }=\sqrt{\frac{G m^{3}}{c^{3} t}}=\sqrt{\frac{m^{3}}{M}}
$$

where also we have considered Equation (6) for the Universe's Mass at Time $t$. According to both equations, obviously we can express minimum energy as: $E_{\min }=m_{\min } \cdot c^{2}$.

\subsubsection{Discrete Energy Variation}

According to Equation (29), any mass/energy variation of material particle o system (set of them), that is: $\Delta E=\Delta m \cdot c^{2}$, can be expressed as a multiple of minimum energy $E_{\min }=m_{\min } \cdot c^{2}$, that is:

$$
\Delta E=n_{q} \cdot\left(m_{\min } \cdot c^{2}\right) \Rightarrow \Delta E=n_{q} \cdot \sqrt{\frac{G m^{3} c}{t}}
$$

where $\left(n_{q} \in \mathbb{Z}\right)$, since $n_{q}$ can be positive or negative if particle or system (set of them) increases or loses energy. For example: By Equation (24) any material particle of the Universe must lose an unrecoverable quantity of mass/energy as Time progresses, which we can express it according to Equation (9), Equation (10) and Equation (29) as:

$$
\Delta E=-\frac{m c^{2}}{t} \Delta t=-\frac{m c^{2}}{\sqrt{N} \cdot \tau} \cdot(n \cdot \tau) \Rightarrow \Delta E=-n \cdot\left(m_{\min } \cdot c^{2}\right)
$$

\subsubsection{Minimum Frequency}

By Equation (26) we can express now the minimum frequency at Time $t$ as:

$$
v=\sqrt{N} \cdot v_{\min } \Leftrightarrow v_{\min }=\sqrt{\frac{G m^{3} c}{h^{2} t}}
$$

\subsection{Discrete Linear Momentum. Minimum Linear Momentum}

Let us now consider any relative reference system. The linear momentum $\boldsymbol{p}$ for any material particle at Time $t$ is defined as: $\boldsymbol{p}=m \boldsymbol{v}$, where $(m, v)$ are the mass and velocity of material particle respectively at Time $t$. However, according to Equation (16) for discrete velocity, we can express the discrete linear momentum $p$ on any material particle $m$ at Time $t$ as:

$$
p=m v \Rightarrow p=n_{v} \cdot\left(m \cdot v_{\text {min }}\right) \Rightarrow p=n_{v} \cdot \sqrt{\frac{G m^{3}}{c t}}
$$

where $\left(n_{v} \in \mathbf{Z}^{+}\right)$is a positive integer. Therefore, the discrete minimum linear momentum $p_{\min }$ of any material particle $m$ at Time $t$ will can be expressed as: 


$$
p_{\min }=m \cdot v_{\min }=m \cdot \frac{l}{t} \Rightarrow p_{\min }=\sqrt{\frac{G m^{3}}{c t}}
$$

Thus, according to previous equation, if we now express $\left(p_{i}=n_{v_{i}} \cdot \frac{m_{i} l}{t_{i}}\right)$ and $m_{i}$ as the particle's linear momentum and particle's mass at Time $t_{i}$ respectively, where $\left(n_{v_{i}} \in \mathbb{Z}^{+}\right)$, then any linear momentum variation $\Delta p$ of the material particle during elapsed Time $\Delta t=t-t_{i}$ can be expressed as:

$$
\Delta p=p-p_{i} \Rightarrow \Delta p=n_{p} \cdot p_{\min } \Leftrightarrow \Delta p=n_{p} \cdot \sqrt{\frac{G m^{3}}{c t}}
$$

With $\left(n_{p} \in \mathbb{Z}\right)$ as a integer which will be positive + or negative - depending if linear momentum variation increases or decreases and where $n_{p}=n_{v}-n_{v_{i}}\left(\frac{t}{t_{i}}\right)^{2}$ since by Equation (23) we have that: $m \cdot t=m_{i} \cdot t_{i} \equiv c t e$.

\subsection{Fractional Orbital Angular Momentum}

In a simple way, quantum mechanics establishes that the angular orbital momentum $L$ of a material particle of mass $m$ is an integer multiple of Planck constant, since the circumference's length must be multiple of the wavelength $\lambda=\frac{h}{m v}$ associated with particle Equation (27), that is:

$$
2 \pi r=n \cdot \frac{h}{m v} \Rightarrow \boldsymbol{L}=\boldsymbol{p} \cdot \boldsymbol{r}=n \cdot \hbar
$$

where $r$ is the distance of the particle from the axis and $p=m v$ the linear momentum at Time $t$ according to Equation (32). Let us now consider any relative reference system. According to Equation (11) for the fundamental minimum length $l$ and Equation (12) for any proper interval of space $\Delta r$, we must express the wavelength and the length of the circumference as multiples of the fundamental minimum length, that is:

$$
2 \pi r=n_{r} \cdot l \wedge \frac{h}{m v}=n_{\lambda} \cdot l
$$

where $\left[\left(n_{r}, n_{\lambda}\right) \in \mathbf{Z}^{+}\right]$are positive integers. Therefore, if we take the variable $I$ from one any equation and replacing it in another, we obtain:

$$
2 \pi r=\left(\frac{n_{r}}{n_{\lambda}}\right) \frac{h}{m v} \Rightarrow \boldsymbol{L}=\boldsymbol{p} \cdot \boldsymbol{r}=\left(\frac{n_{r}}{n_{\lambda}}\right) \cdot \hbar
$$

where we verify that orbital angular momentum of any material particle at Time $t$, including photons of light, is not an integer but a fraction of reduced Planck's constant, as has already been demonstrated in [4] by Kyle E. Ballantine et al., that describe that in reduced dimensions, photons can have a half-integer total angular momentum.

\subsection{Fractional Force $\boldsymbol{F}$ Acting on a Material Particle}

Let us now consider any relative reference system. The force $\boldsymbol{F}$ acting on a 
material particle at Time $t$ can be expressed as:

$$
\boldsymbol{F}=\frac{\Delta \boldsymbol{p}}{\Delta t}=\frac{\Delta}{\Delta t}(m \boldsymbol{v})
$$

where $\boldsymbol{p}=m \boldsymbol{v}$ is the linear momentum at Time $t$ according to Equation (32). By Equation (24) any material particle of the Universe must lose an unrecoverable part of mass/energy as Time progresses, thus we obtain:

$$
F=\left(\frac{\Delta m}{\Delta t} v+m \frac{\Delta v}{\Delta t}\right)=-m \frac{v}{t}+m a \Rightarrow F=m a\left(1-\frac{v}{a t}\right)
$$

where $a=\frac{\Delta v}{\Delta t}$ is the fractional acceleration at Time $t$ of the material particle according to Equation (18). However, we can consider $v_{i}=0$ at Time $t_{i}$ and by the relation between acceleration and velocity at Time $t$ is: $H(t)=\frac{a}{v}=\frac{1}{\Delta t}$ according to Equation (21), we finally obtain the Force $F$ acting on a material particle at Time $t$ as:

$$
F=m a\left(1-\frac{\Delta t}{t}\right) \Rightarrow F=m \cdot a(t) \Leftrightarrow a(t)=a\left(1-\frac{\Delta t}{t}\right)
$$

Therefore, any force in Nature decreases as Time progresses and where $m$ and $a(t)$ are defined at Time $t$ respectively. Note that is only valid when there is a elapsed proper time interval $\Delta t$. If we determine, by measurement for example, the mass $m$ and acceleration $a$ at Time $t$ respectively, then $\Delta t=0$ and $a(t)=a$ with $F=m a$, but its evolution as Time progresses will be done by Equation (35).

Thus, fractional force $F$ can be generally expressed according to Equation (18) for the fractional acceleration $a$ at Time $t$ of the material particle, as:

$$
F=\left(\frac{n_{\alpha}}{n}\right) \cdot \frac{m c}{t}\left(1-\frac{\Delta t}{t}\right)
$$

where $\left(n \in \mathbb{Z}^{+}\right)$and $\left(n_{\alpha} \in \mathbb{Z}\right)$.

\subsection{Universe Energy and Mass}

Let us now consider any relative reference system. According to Equation (4) and Equation (29) for de minimum mass, we can express de Universe's Mass at Time $t$ as:

$$
M=N \cdot m=N \sqrt{N} \cdot m_{\min } \Rightarrow M=\aleph \cdot m_{\min } \Leftrightarrow \aleph=N \sqrt{N}
$$

And therefore the Universe's Energy at Time $t$ as:

$$
E_{U}=N \cdot m c^{2}=N \sqrt{N} \cdot m_{\min } c^{2} \Rightarrow E_{U}=\aleph \cdot m_{\min } c^{2} \Leftrightarrow \aleph=N \sqrt{N}
$$

where $\aleph=N \sqrt{N}$ is the total minimum masses number or can be also understood as the total information bits number. Also, according to Equation (26) and Equation (31), we can now the Universe's Energy at Time $t$ as:

$$
E_{U}=N \cdot h v=N \sqrt{N} \cdot h v_{\min } \Rightarrow E_{U}=\aleph \cdot h v_{\min } \Leftrightarrow \aleph=N \sqrt{N}
$$

That is, Universe's Energy as an ensemble of $\aleph=N \sqrt{N}$ quantum oscillators. 


\subsection{Universe's Area and Holographic Principle}

According to Equation (8) and Equation (29) we can express at Time $t$ the number $\aleph$ of information bits or minimum masses as:

$$
N=\frac{c^{3} t}{G m}=\frac{c^{3}}{\hbar G}\left(\frac{\hbar t}{m}\right)=\frac{c^{3}}{\hbar G}\left(\frac{\hbar t}{\sqrt{N} \cdot m_{\min }}\right) \Rightarrow \aleph=N \sqrt{N}=\frac{c^{3}}{\hbar G}\left(\frac{\hbar t}{m_{\min }}\right)
$$

where the quantity $\left(\frac{\hbar t}{m_{\min }}\right)$ has dimensions of Area $\left(\mathrm{m}^{2}\right)$ according to holographic principle proposed by Professor Gerard 't Hooft [5]. Therefore, by Equation (8) and Equation (5) we can express the Universe's Area $A$ as a function of $N \sqrt{N}$ information bits or minimum masses as:

$$
\aleph=\frac{c^{3} A}{\hbar G} \Leftrightarrow A=\left(\frac{\hbar t}{m_{\text {min }}}\right)=\frac{\hbar t}{m} \sqrt{N}=\sqrt{\frac{\hbar^{2} t^{3} c^{3}}{G m^{3}}}=\frac{c^{2} t^{2}}{\sqrt{\Omega}}
$$

Obviously, we can also express $\aleph=N \sqrt{N}$ at Time $t$ as a set of $n$ subsystems, as:

$$
\aleph=\sum_{j=1}^{n} \aleph_{j}=\aleph_{1}+\aleph_{2}+\cdots+\aleph_{i}+\cdots+\aleph_{n}
$$

Thus, any subsystem of $\left(\aleph_{i} \subset \aleph_{;} \aleph_{i} \in \mathbb{Z}^{+}\right)$minimum masses or information bits within the Universe can be expressed at Time $t$ as:

$$
\aleph_{i}=\frac{\aleph}{\beta_{i}} \Leftrightarrow \beta_{i}=1+\frac{1}{\aleph_{i}}\left(\sum_{j=1}^{i-1} \aleph_{j}+\sum_{j=i+1}^{n} \aleph_{j}\right)
$$

Therefore, any system of $\aleph_{i}$ information bits or minimum masses within the Universe with area $A_{i}=\frac{A}{\beta_{i}}$ and total mass $M_{i}=\aleph_{i} \cdot m_{\min }$ by Equation (37) and Equation (41), can be expressed by Equation (40) at Time $t$ as:

$$
\aleph_{i}=\frac{c^{3} A_{i}}{\hbar G}
$$

\section{Thermodynamical Part}

\subsection{Expanding Universe as Consequence of a Particle Creation Process as an Alternative to Dark Energy}

Consider with regard to a relative reference system any interval of proper time $\Delta t=t-t_{i}$ according to Equation (10). By Equation (6) the Universe Mass must increase as Time progresses according to Passage of Time Principle. Therefore if the Universe Mass at initial Universe Time $t_{i}$ is $M\left(t_{i}\right)$ and by Passage of Time Principle, the Universe Mass at any later Universe Time $t$ is $M(t)$, then the increase of Universe's Mass $\Delta M=M(t)-M\left(t_{i}\right)$ during elapsed interval of proper time $\Delta t=t-t_{i}$ will be:

$$
\Delta M=\frac{c^{3}}{G} \Delta t \Rightarrow \Delta E_{U}=\Delta M c^{2}=\frac{c^{5}}{G} \Delta t
$$

where we can establish that $E_{U}=M c^{2}=N \cdot m c^{2}$ is the Universe Energy at Time $t$ according to Equation (38). 
However, we know that $\Delta t=n \cdot \tau$ by Equation (10), then by Equation (7) for fundamental minimum time and according to Equation (8) for the total number of particles $N$, we can express the previous equation as:

$$
\Delta E_{U}=n \cdot \frac{c^{5}}{G} \sqrt{\frac{G m t}{c^{3}}} \Rightarrow \Delta E_{U}=n \cdot \sqrt{N} \cdot m c^{2}
$$

Therefore according to Passage of Time Principle, the Universe increases its Mass/Energy as Time progresses by the creation of $n \sqrt{N}$ new particles of mass $m$ and energy $m c^{2}$ at every proper time interval $\Delta t$, or what is the same, by the creation of $\sqrt{N}$ new particles of mass $m$ and energy $m c^{2}$ at every fundamental minimum time $\tau$.

However, according to the first principle of Thermodynamics, near the equilibrium any thermodynamic system at temperature $T$ experiences a state change according to equation:

$$
\Delta E=T \Delta S+\Delta W_{e x t}
$$

where $\Delta E$ is the energy variation of the system, $T \Delta S$ is the work done by internal forces generating a change of entropy $\Delta S$ and $\Delta W_{\text {ext }}$ is the work done by external forces acting on the system.

However, by passage of Time principle the mass/energy of the Universe increases as Time progresses according to Equation (44), thus we can consider the Universe at every Time $t$ as a entropic system, that is, the universe entropy is a function of its internal energy and consequently can exist no external forces acting on it, that is: $\Delta W_{e x t}=0$. Applying the first principle, the increase of Energy of the Universe $\Delta E_{U}$ can be expressed then as:

$$
\Delta E_{U}=T \Delta S \Leftrightarrow \Delta W_{e x t}=0
$$

However, by Equation (8) for the total number of particles $N$, Equation (5) for the value of $\Omega$ at Universe Time $t$, Equation (7) for fundamental minimum time $\tau$, Equation (9) for Universe Time and Boltzmann constant $k_{B}$ which relates the thermodynamic energy and temperature, we can express Equation (44) for the increase of energy of the Universe $\Delta E_{U}$ as:

$$
\Delta E_{U}=n \cdot \sqrt{N} \cdot m c^{2}=T \Delta S \Rightarrow \Delta E_{U}=\frac{\hbar c^{3}}{k_{B} G m} \cdot n k_{B} \sqrt{\frac{m^{3} c G t}{\hbar^{2}}}
$$

Therefore we obtain by equalizing:

1)

$$
T=\frac{\hbar c^{3}}{k_{B} G m}
$$

The temperature or thermal radiation $T$ associated to the increase of Energy of the Universe $\Delta E_{U}$ by the creation of $n \sqrt{N}$ new particles of mass $m$ and energy $m c^{2}$ at every proper time interval $\Delta t$.

2)

$$
\Delta S=n \cdot k_{B} \sqrt{\frac{m^{3} c G t}{\hbar^{2}}} \Rightarrow \Delta S=k_{B} \frac{m c^{2}}{\hbar} \Delta t
$$

The entropy increase $\Delta S$ on every material particle $m$ during proper time 
interval elapsed $\Delta t=t-t_{i}=n \cdot \tau$ according Equation (10) and fundamental minimum time Equation (7).

\section{Accelerated Universe}

According to Mechanics we know that the work done by any force $\boldsymbol{F}$ on a material body produces a variation of its energy, where the work can be expressed as the product of force for the displacement done, that is:

$$
\Delta E=\boldsymbol{F} \cdot \Delta \boldsymbol{r}
$$

Therefore, if we establish that the increase of Universe energy $\Delta E_{U}$ according Equation (46) is due to a force $F$ of creation of $n \sqrt{N}$ new particles of mass $m$ and energy $m c^{2}$ during proper time interval $\Delta t$ elapsed producing a displacement $\Delta R$, which can be considered as the increase of Radius ${ }^{4}$ of the Universe $R$ at the same proper time interval $\Delta t$, then $\Delta E_{U}$ can be expressed as:

$$
\Delta E_{U}=F \Delta R=T \Delta S \Rightarrow F \Delta R=\frac{\hbar c^{3}}{k_{B} G m} \cdot k_{B} \frac{m c^{2}}{\hbar} \Delta t=\frac{c^{5}}{G} \Delta t
$$

However, according to Equation (12) we can express $\Delta R=n_{R} \cdot l$ and by Equation (10), $\Delta t=n \cdot \tau$ with $l=c \cdot \tau$ and where $\left(n_{R}, n\right)$ are positive integers. Therefore we can obtain the force $F$ as:

$$
F=\left(\frac{n}{n_{R}}\right) \cdot \frac{c^{4}}{G}
$$

That is, the force $F$ of creation of new particles of mass $m$ during proper time interval $\Delta t$ elapsed as a fraction of Planck force, $F_{P}=\frac{c^{4}}{G}$. Now, according to Equation (44) and Equation (50) we can express Universe's Radius increase $\Delta R$ as:

$$
\Delta R=n_{R} \cdot \frac{G}{c^{4}}\left(\sqrt{N} \cdot m c^{2}\right)
$$

That is, as a multiple of Universe energy increase by the creation of $\sqrt{N}$ new particles of mass $m$ and energy $m c^{2}$ at every fundamental minimum time $\tau$.

However, considering: $v_{U}=\frac{\Delta R}{\Delta t}$ as velocity of expansion of the Universe according to Equation (16), $a_{U}=\frac{\Delta v_{U}}{\Delta t}$ the Universe's acceleration according to Equation (18), $F=\frac{\Delta p_{U}}{\Delta t}$ according to Equation (34) and $p_{U}=M v_{U}$ as

Universe's linear momentum at Time $t$, then we can obtain according to Equation (49) the force $F$ at Time $t$ as:

$$
F=M \frac{c^{2}}{v_{U} t} \Rightarrow M \frac{c^{2}}{v_{U} t}=\frac{\Delta p_{U}}{\Delta t} \Rightarrow M \frac{c^{2}}{v_{U} t}=\frac{\Delta M}{\Delta t} v_{U}+M \frac{\Delta v_{U}}{\Delta t}
$$

Thus, simplifying according to Equation (6) for Universe's Mass we obtain:

${ }^{4}$ It is logical to think that due to the creation of $n \sqrt{N}$ new particles of mass $m$ at every proper time interval $\Delta t$ according Equation (44), the volume of the Universe must increase and therefore its Radius $R$, since otherwise and due to Gravity, the Universe would collapse in a sufficiently large Time. 


$$
v_{U}\left(v_{U}+a_{U} t\right)=c^{2} \Rightarrow\left\{\begin{array}{l}
a_{U}=\frac{c^{2}}{v_{U} t}\left(1-\frac{v_{U}^{2}}{c^{2}}\right) \\
v_{U}=\frac{c}{\sqrt{1+H(t) \cdot t}} \\
H(t)=\frac{1}{t}\left(\frac{c^{2}}{v_{U}^{2}}-1\right)
\end{array}\right.
$$

where $H(t)=\frac{a_{U}}{v_{U}}$ according to velocity-distance law Equation (21). We can easily verify that $v_{U} \leq c$ according to relativity and we prove that $v_{U} \neq 0$. Also we can obtain that: $\left(v_{U}=c \Leftrightarrow a_{U}=0\right)$. Thus, we can prove that the origin of the acceleration of the Universe is a consequence of particles creation as Time progresses as an alternative to dark energy. However, also Dark Energy (the acceleration of the Universe) could be, in principle, solved through the extended theories of gravity [6].

\subsection{Universe's Radius}

Let us now consider any relative reference system. The increase of Universe's Energy by Equation (43) can be now expressed according to Equation (6) as:

$$
\Delta E_{U}=\frac{M c^{2}}{t} \Delta t
$$

Also, Equation (47) for the temperature/thermal radiation $T$ associated to the increase of Universe's Energy $\Delta E_{U}$ can be now expressed according to Equation (8) as:

$$
T=\frac{\hbar c^{3}}{k_{B} G m} \Rightarrow T=N \cdot T_{m} \Leftrightarrow T_{m}=\frac{\hbar}{c k_{B}}\left(\frac{c}{t}\right)
$$

where $T_{m}$ can be considered as the temperature/thermal radiation associated to the material particle at Time $t$. Thus, according to Equation (29) for minimum mass, Equation (53) and Equation (48), we can now express $\Delta E_{U}=T \Delta S$ as:

$$
\frac{M c^{2}}{t} \Delta t=N \cdot T_{m} \cdot k_{B} \frac{m c^{2}}{\hbar} \Delta t \Rightarrow M c^{2}=N \sqrt{N} k_{B} T_{m}\left(\frac{m c^{2} t}{\hbar \sqrt{N}}\right)
$$

Therefore, by Equation (5) for $\Omega$, Equation (8), Equation (40) for $\aleph=N \sqrt{N}$ and by applying the equipartition theorem of energy, we obtain:

$$
M c^{2}=\sqrt{\Omega} \cdot \aleph k_{B} T_{m} \Rightarrow M c^{2}=\frac{1}{2} \aleph k_{B} T_{m} \Leftrightarrow \Omega=\frac{1}{4}
$$

Thus, according to Equation (40) we obtain for Universe's Area:

$$
A=\frac{c^{2} t^{2}}{\sqrt{\Omega}} \Rightarrow A=2 c^{2} t^{2}
$$

However, we can express in a simple way the area $A_{i}$ of any object as a function of its radius $r$ as: $A_{i}=f \cdot r^{2}$, where $\left(f \in \mathbb{R}^{+}\right)$is a real positive number. Therefore, we can obtain for the Universe's Area $A$ as a function of Universe's Radius $R$ at Time $t$ as: 


$$
A=f \cdot R^{2}=2 c^{2} t^{2} \Rightarrow R=c t \quad \Leftrightarrow \quad f=2
$$

where we have applied the principle of simplicity. Thus, if we replace the value for the fundamental minimum length $(l=c \tau)$ in Equation (9) we obtain the equation:

$$
c t=\sqrt{N} \cdot l \Rightarrow R=\sqrt{N} \cdot l
$$

which is the well-known Weyl-Eddington relationship.

\subsection{Universe's Entropy and Time's Arrow}

We can also express the increase of Universe Energy $\Delta E_{U}=T \Delta S$ during elapsed interval of proper time $\Delta t$ by Equation (52) and Equation (53), as:

$$
\frac{c^{5}}{G} \Delta t=N \cdot \frac{\hbar}{c k_{B}}\left(\frac{c}{t}\right) \cdot \Delta S \Rightarrow S_{U}=\frac{k_{B} c^{5} t}{\hbar G} \Delta t \quad \Leftrightarrow \quad S_{U}=N \cdot \Delta S
$$

Establishing $S_{U}$ as the total Universe's entropy during elapsed interval of proper time $\Delta t$. However, if we consider "Universe's Time as the total time elapsed", that is: $\Delta t=t$, then we obtain the total Universe's entropy at Time $t$ as:

$$
S_{U}=\frac{k_{B} c^{5}}{\hbar G} t^{2}
$$

Thus, by passage of Time principle the Universe's Entropy increases as Time progresses and we can explain why the Universe had less entropy in the past, resulting in a clear distinction between past and future. Approaching $t_{0} \simeq \frac{1}{H_{0}}$, the value $H_{0}=(74.03 \pm 1.42) \mathrm{km} \cdot \mathrm{s}^{-1} \cdot \mathrm{Mpc}^{-1}$ in [7], we obtain: $S_{U} \sim 10^{122} k_{B}$.

On the other hand, Professor Bekenstein [8] has proposed the existence of a universal upper bound on the entropy-to-energy ratio $S_{B}$ for any object of maximal radius $R$ and total energy $E$, as:

$$
S_{B}=\frac{2 \pi k_{B} R E}{\hbar c}
$$

Therefore, according to Equation (56) for the Universe's Radius and $E_{U}=M c^{2}$ with $M$ in Equation (6), we can express the total Universe's Entropy Equation (58) as:

$$
S_{U}=\frac{k_{B} R E_{U}}{\hbar c} \Rightarrow S_{U}<S_{B}
$$

which is in perfect agreement with the universal bound on the entropy.

\subsubsection{Universe's Entropy as a Function of Universe's Area}

Equation (58) can be also expressed according to Equation (55) for the Universe's area $A=2 c^{2} t^{2}$, as:

$$
S_{U}=\frac{k_{B} c^{3}}{2 \hbar G} A
$$

which establish Universe's Entropy as a function of Universe's Area. 


\subsubsection{Universe's Entropy as a Function of Total Minimum Masses Number}

According to Equation (40) for the total information bits number or total minimum masses number as a function of Universe's Area, that is: $\aleph=\frac{c^{3} A}{\hbar G}$, we can now express Universe's Entropy Equation (59) as:

$$
S_{U}=\aleph \cdot \frac{k_{B}}{2}
$$

\subsection{Entropy-Action Equivalence Law}

Let us now consider any relative reference system. According to Equation (48) any particle (or system) of total mass $m$ at Time $t$ increase its entropy during the proper time interval elapsed $\Delta t=t-t_{i}$ as:

$$
\Delta S=k_{B} \frac{m c^{2}}{\hbar} \Delta t \Leftrightarrow \Delta S=\frac{k_{B}}{\hbar}(E \cdot \Delta t)
$$

which we can establish as law of the entropy increase for any material particle during proper time interval elapsed $\Delta t$ or also entropy-action equivalence law, since as we verify the increase of entropy $\Delta S$ is proportional to the action $E \cdot \Delta t$.

\subsubsection{Energy vs. Time Interval Relationship}

An important result is the relationship between the energy $E=m c^{2}$ of a material particle at Universe's Time $t$ and the time interval $\Delta t$ in which can be measured. By Equation (7) for fundamental minimum time and Equation (54) we have:

$$
m c^{2} \cdot \tau=\sqrt{\Omega} \cdot \hbar \Rightarrow E \cdot \tau=\frac{\hbar}{2}
$$

Now, multiplying by $n \in \mathbb{Z}^{+}$for any proper time interval according to Equation (10) we obtain:

$$
E \cdot \Delta t=n \cdot \frac{\hbar}{2} \Rightarrow E \cdot \Delta t \geq \frac{\hbar}{2}
$$

which explains the impossibility of measuring the energy of a material particle at any given time interval $\Delta t<\tau$.

\subsubsection{Discrete Entropy and Time's Arrow}

According to Equation (61) and Equation (62) we can also obtain discrete increase of entropy $\Delta S$ as:

$$
\Delta S=n \cdot \frac{k_{B}}{2}
$$

Thus, discrete entropy increase is a multiple of $\frac{k_{B}}{2}$. Obviously $\frac{k_{B}}{2}$ represents the minimum production of entropy. Equation (63) also can be expressed as:

$$
\Delta S \geq \frac{k_{B}}{2} \Rightarrow \Delta S>0
$$


which represents the arrow of time for any material particle or set of them (system).

\subsubsection{Special Relativistic Entropy-Action Equivalence Law}

According to Equation (4), the total Mass of the Universe $M$ at Universe's Time $t$ can be expressed as the ensemble of $N$ typical particles in motion of mass $m$. If the elapsed proper time interval $\Delta t$ is small enough, we can consider the velocity $v$ of material particle in motion as constant. Thus, according to special relativity we know that:

$$
m=\frac{m_{0}}{\sqrt{1-\frac{v^{2}}{c^{2}}}} \Rightarrow m c^{2}=\sqrt{p^{2} c^{2}+m_{0}^{2} c^{4}}
$$

where $m$ and $m_{0}$ are particle's mass in motion and rest respectively at Time $t$ and $p=m v$ as lineal momentum at Time $t$. Therefore, by Equation (61) we can now express the special relativistic entropy-action equivalence law as:

$$
\Delta S=k_{B} \frac{m c^{2}}{\hbar} \Delta t=\sqrt{\left(k_{B} \frac{m c}{\hbar} \Delta r\right)^{2}+\left(k_{B} \frac{m_{0} c^{2}}{\hbar} \Delta t\right)^{2}}
$$

where $\Delta r=v \Delta t$ is the displacement during elapsed proper time interval $\Delta t$.

Therefore, if we denote $\Delta S_{k}=k_{B} \frac{m c}{\hbar} \Delta r$ as the kinetic entropy due to motion and $\Delta S_{0}=k_{B} \frac{m_{0} c^{2}}{\hbar} \Delta t$ as entropy at rest during elapsed proper time interval $\Delta t$, we can express also the special relativistic entropy-action equivalence law as:

$$
\Delta S=\sqrt{\left(\Delta S_{k}\right)^{2}+\left(\Delta S_{0}\right)^{2}}
$$

\subsection{Fractional Temperature}

In classical Thermodynamics, for a system (particle or set of them) of mass $m$, where entropy $S$ is a function of its internal energy $E$, the temperature $T$ at Time $t$ is given by:

$$
\frac{1}{T}=\frac{\Delta S}{\Delta E}
$$

However, according to Equation (5), Equation (29), and $\Omega=\frac{1}{4}$ by Equation (54) we can express the minimum mass $m_{\min }$ at Time $t$ as:

$$
m_{\min }=\frac{\hbar}{2 c^{2} t}
$$

Therefore, by Equation (30) we have proved that any energy's variation can be expressed as multiple of $E_{\min }=m_{\min } \cdot c^{2}$ and Equation (63) for discrete entropy, we can now express the temperature as:

$$
T=\frac{\Delta E}{\Delta S}=2\left(\frac{n_{q}}{n}\right) \cdot \frac{m_{\min } c^{2}}{k_{B}}=2\left(\frac{n_{q}}{n}\right) \cdot \frac{\hbar c^{2}}{2 k_{B} c^{2} t} \Rightarrow T=\left(\frac{n_{q}}{n}\right) \cdot \frac{\hbar}{k_{B} t}
$$

which represents the fractional temperature $T$ at Time $t$ where $\left(n_{q} \in \mathbb{Z}\right)$ and 
$\left(n \in \mathbb{Z}^{+}\right)$.

Temperature as a Function of Acceleration

We can also express Equation (65) as:

$$
T=\frac{\hbar}{c k_{B}} \cdot\left(\frac{n_{q}}{n}\right) \frac{c}{t}
$$

However, according to Equation (18) for fractional acceleration of any particle or system, we now obtain:

$$
T=\frac{\hbar a}{c k_{B}} \Leftrightarrow a=\left(\frac{n_{q}}{n}\right) \frac{c}{t}
$$

where obviously the term $\left(\frac{n_{q}}{n}\right) \frac{c}{t}$ is equal to an acceleration according to Equation (18). Therefore, any material particle with acceleration a have associated a temperature o thermal radiation $T$ according to Equation (66).

\section{Gravitational Part}

\subsection{Universe Gravitational Potential}

By Equation (6) for the Universe's Mass and Equation (56) for the Universe's Radius, we obtain:

$$
\frac{G M}{R}=c^{2}
$$

Therefore, we can now express the Universe gravitational Potential $\Phi$ at Time $t$ as:

$$
m c^{2}+m \Phi=0 \Leftrightarrow \Phi=-\frac{G M}{R}
$$

That is, there exists a non-local collective gravitational interaction of all particles within the Universe's Horizon, as a consequence of which all $\mathrm{N}$ typical particles of mass $m$ in motion in the Universe are "gravitationally entangled" and form a unified statistical ensemble.

Thus, we can relate the origin of inertia of any material particle to its interactions with the whole universe, according to the non-local potential of the whole universe, $\Phi$, acting on any material particle of the world ensemble as:

$$
m c^{2}=-m \Phi \quad \Leftrightarrow \quad m c^{2}=\frac{G M m}{R}
$$

where $E=m c^{2}$ is the total energy of the particle at Universe Time $t$ according to Equation (26). Therefore also we can now express the origin of $v$ as a fundamental frequency of oscillation associated with every particle $m$ at Time $t$ due its interactions with the whole universe, according to the non-local potential of the whole universe, $\Phi$, acting on any material particle as:

$$
h v=-m \Phi \quad \Leftrightarrow \quad h v=\frac{G M m}{R}
$$




\subsection{On the Origin of Gravity}

Let us now consider any relative reference system. By Equation (52) and Equation (53), we can now express the increase of Universe Energy $\Delta E_{U}=T \Delta S$ during elapsed proper time interval $\Delta t$ according to Equation (49) as:

$$
\frac{M c^{2}}{t} \Delta t=N \cdot T_{m} \cdot k_{B} \frac{m c^{2}}{\hbar} \Delta t
$$

However, by Equation (54) for the equipartition theorem of energy for the Universe, Equation (40) for the number $\aleph=\frac{c^{3} A}{\hbar G}$ and Equation (56) for Universe's Area at Time $t$, that is: $A=2 c^{2} t^{2}=2 R^{2}$, we obtain:

$$
M c^{2}=\frac{1}{2} \frac{c^{3}}{\hbar G}\left(2 R^{2}\right) k_{B} T_{m} \Rightarrow T_{m}=\frac{\hbar}{c k_{B}}\left(\frac{G M}{R^{2}}\right)
$$

which is according to Equation (66) for temperature as a function of acceleration. Then, by substitution of $T_{m}$ in Equation (67), by Equation (4) and simplifying, we obtain:

$$
-\frac{m c^{2}}{t} \Delta t=-\frac{G M m}{R^{2}} \cdot c \Delta t
$$

However, by Equation (9), fundamental minimum length $l=c \tau$, Equation (12) for any length and Equation (29) for minimum mass, this previous equation can also be expressed as:

$$
-m_{\min } c^{2}=-\frac{G M m}{R^{2}} \cdot l \quad \Rightarrow \quad-n \cdot m_{\min } c^{2}=-\frac{G M m}{R^{2}} \cdot \Delta r \Leftrightarrow \Delta r=n \cdot l
$$

where obviously $\left(n \in \mathbf{Z}^{+}\right.$). Therefore, a consequence of that all $N$ typical particles in the universe are "gravitationally entangled" is that the work done by the gravitational force $\left(F_{G}=-\frac{G M m}{R^{2}}\right)$ of whole Universe acting on every material particle during elapsed proper time interval $\Delta t$ causes an unrecoverable loss of energy $\Delta E=-n \cdot m_{\min } c^{2}$, that is, the needed energy to displace a material particle of the world ensemble to the distance $\Delta r$.

Or also we can express that every unrecoverable lost minimum mass $m_{\min }$ is due the work done by $\left(F_{G}=-\frac{G M m}{R^{2}}\right)$ the Universe gravitational force acting on any material particle at every $\tau$, that is, as the energy needed to displace a particle of the world ensemble to the fundamental minimum length $l$.

\section{Negative Acceleration}

By Equation (64) we can now express the quantity of energy lost at Time $t$ during elapsed proper time interval $\Delta t$ according to Equation (68) as:

$$
\Delta E=-n \cdot m_{\min } c^{2}=-n \cdot \frac{\hbar}{2 t} \Rightarrow-n \cdot \frac{\hbar}{2 t}=-\frac{G M m}{R^{2}} \cdot \Delta r
$$

Obviously since we have considered the Universe as an entropic system Equa- 
tion (45), this quantity of energy lost for every material particle at Time $t$ can be expressed as: $\Delta E=T \Delta S$. Therefore according to Equation (66) for temperature as a function of acceleration and Equation (63) for discrete entropy, we obtain:

$$
\frac{\hbar}{k_{B} c}\left(-\frac{c}{t}\right) \cdot n\left(\frac{k_{B}}{2}\right)=-\frac{G M m}{R^{2}} \cdot \Delta r
$$

Therefore, we obtain that the work done by the Universe gravitational force $F_{G}=-\frac{G M m}{R^{2}}$ causes a negative acceleration $a_{P}=-\frac{c}{t}$ on every material particle due to the loss of every minimum mass $m_{\min }$ as Time progresses. According to Equation (20) we can express as:

$$
\left[a_{P}=-\frac{c}{t} \Rightarrow a_{P}=-\left(\frac{n_{r}}{n_{v}}\right) c H(t)\right]
$$

where $\left(n_{v}, n_{r}\right)$ are any positive integers. This negative acceleration should be perceived in those objects or particles not subjected to any other force.

\subsection{Quantum Gravity}

According to $₫ 5.1$ we found that exists a non-local collective gravitational interaction of all particles within the Universe's Horizon, as a consequence of which all $\mathrm{N}$ typical particles of mass $m$ in motion in the Universe are "gravitationally entangled" and form a unified statistical ensemble.

Thus, according to Equation (37) and Equation (41) we will consider any local subsystem within the Universe of $\aleph_{i}$ minimum masses or $N_{i}$ material particles of total mass $M_{i}$, that is: $M_{i}=N_{i} \cdot m=\aleph_{i} \cdot m_{\min }$, that form a closed surface of area $A_{i}$ at Time $t$. For simplicity, we will consider that the total mass $M_{i}$ is concentrated in the center of the surface. We will also consider that a material particle or body of mass $m$ is located on the surface at a distance $r$ from the center of the surface at Time $t$ and that there is no external force acting on the subsystem or on the material particle, thus the total local system is isolated.

\subsubsection{Equivalence's Principle as a Consequence of Discrete Space}

Now, according to Equation (12) for the discrete space, we can now express at Time $t$ the distance $r$ from the center of surface which is located the material particle or body of mass $m$ as:

$$
r=n_{r} \cdot l \Rightarrow r=n_{r} \cdot \sqrt{\frac{G m t}{c}} \Rightarrow r^{2}=n_{r}^{2} \cdot \frac{G m t}{c}
$$

Obviously, this length $r$ also can be expressed by Equation (57) as a function of Universe radius $R=\sqrt{N} \cdot l$ as:

$$
\frac{r}{R}=\frac{n_{r}}{\sqrt{N}} \Rightarrow r=\left(\frac{n_{r}}{\sqrt{N}}\right) R
$$

However, the system have total mass $M_{i}=N_{i} \cdot m$, thus, substituting $m$ in Equation (71) we obtain: 


$$
r^{2}=\left(\frac{n_{r}^{2}}{N_{i}}\right) \frac{G M_{i} t}{c} \Rightarrow \frac{G M_{i}}{r^{2}}=\left(\frac{N_{i}}{n_{r}^{2}}\right) \frac{c}{t}
$$

However, we found that $a=\left(\frac{N_{i}}{n_{r}^{2}}\right) \frac{c}{t}$ is equal to a fractional inertial acceleration according to Equation (18). Therefore we can express the gravitational acceleration at Time $t$ as:

$$
-\frac{G M_{i}}{r^{2}}=-\left(\frac{N_{i}}{n_{r}^{2}}\right) \frac{c}{t} \Rightarrow-\frac{G M_{i}}{r^{2}}=a=\frac{\Delta v}{\Delta t}
$$

Thus, we prove that a gravitational acceleration is equal to an any inertial acceleration, that is, the complete physical equivalence of a gravitational acceleration and a corresponding acceleration of the reference system.

\subsubsection{Emergent Gravity}

The local system is isolated, therefore there is no external force acting on the surface or on the material particle and therefore system entropy $S$ is a function of its internal energy $E$ and the work $T \Delta S$ done by the internal force during any elapsed proper time interval $\Delta t$ can be expressed according to Mechanics as: $F \Delta r=T \Delta S$ where $T$ is the temperature o thermal radiation at Time $t$ associated to the material particle and $F$ is now a entropic force.

Thus we can express, according to Equation (4) for Universe Mass $M=N \cdot m$, the local subsystem mass $M_{i}=N_{i} \cdot m$ and Equation (72) for relation between $r$ and $R$, the work done by the entropic gravitational force $F$ according to Equation (68) $\mathrm{as}^{5}$ :

$$
-n_{i} \cdot m_{\min } c^{2}=-\frac{G M_{i} m}{r^{2}} \cdot \Delta r
$$

where $\left(n_{i}=\frac{n \cdot N_{i}}{n_{r}^{2}} ; n_{i} \in \mathbb{Z}^{+}\right)$according to Equation (30). Therefore, the work done by gravitational force $\left(F=-\frac{G M_{i} m}{r^{2}}\right)$ acting on the material particle $m$ during elapsed proper time interval $\Delta t$, which is located at a distance $r$ from the center of surface, causes an unrecoverable loss of energy $\Delta E=-n_{i} \cdot m_{\min } c^{2}$, that is, the needed energy to displace a material particle to the distance $\Delta r$.

However, this quantity of lost energy by every material particle at Time $t$ can be expressed as: $\Delta E=T \Delta S$. Therefore according to Equation (66) for temperature as a function of acceleration and Equation (73) for the equivalence principle, we can express Equation (74) as:

$$
\frac{\hbar}{k_{B} c}\left(-\frac{G M_{i}}{r^{2}}\right) \cdot \Delta S=-\frac{G M_{i} m}{r^{2}} \cdot \Delta r \Leftrightarrow \Delta S=k_{B} \frac{m c}{\hbar} \Delta r
$$

which it's the Verlinde result in [9].

${ }^{5} M=\left(\frac{N}{N_{i}}\right) M_{i}$ and $r^{2}=\left(\frac{n_{r}^{2}}{N}\right) R^{2}$ and replacing theses values in Equation (68). 


\subsubsection{Graviton Mass as the Fundamental Minimum Mass}

It's remarkable that both in Equation (68) for the Universe gravitational force $F_{G}$ and Equation (74) for any gravitational force $F$, the lost energy $\Delta E$ by every material particle $m$ due to the work done by both forces is a multiple of $m_{\min } c^{2}$. Thus, we postulate that this lost minimum mass in every fundamental minimum time $\tau$ can be considered the mass of "graviton", that is: $m_{\min }=m_{g}$.

Therefore according to Equation (64) we obtain:

$$
m_{g} c^{2}=\frac{1}{2} \hbar w \Leftrightarrow w=\frac{1}{t}
$$

which matches with the ground state (the state with lowest energy) of a quantum harmonic oscillator that has a non vanishing zero-point energy where $w$ is the oscillation frequency (angular frequency). Thus, according to Equation (39) Universe's Energy can be expressed as a ensemble of $\aleph=N \sqrt{N}$ quantum oscillators. Also, graviton energy $E_{g}=\frac{1}{2} \hbar w$, can be expressed according to the known relationship $w=2 \pi v$ and $\hbar=\frac{h}{2 \pi}$, as:

$$
m_{g} c^{2}=\frac{1}{2} h v \Leftrightarrow \quad \Leftrightarrow=\frac{1}{t}
$$

Therefore graviton mass value can be approximated as:

$$
m_{g}=\frac{1}{2} \frac{h}{c^{2} t} \Rightarrow m_{g} \approx \frac{h H_{0}}{2 c^{2}}
$$

Thus, approaching $t_{0} \approx \frac{1}{H_{0}}$, the value $H_{0}=(74.03 \pm 1.42) \mathrm{km} \cdot \mathrm{s}^{-1} \cdot \mathrm{Mpc}^{-1}$ in [7], we can obtain approximately: $m_{g} \sim 10^{-68} \mathrm{~kg}$ which can be in agreement with the range of values $m_{g} \sim\left(10^{-68}-10^{-66}\right) \mathrm{kg}$ for the entropic minimum mass found by the Prof. J. R. Mureika and R. B. Mann in [10] where this range represents the smallest non-zero mass for any particle quanta in the entropic gravity framework.

\subsubsection{Gravitational Entropy}

According to Equation (59), Equation (60), Equation (41) and by Equation (42), the total entropy of any system within the Universe with total mass $M_{i}$ can be expressed as a function of its total minimum masses number $\aleph_{i}$ or its Area $A_{i}$ at Time $t$ as:

$$
S_{i}=\frac{k_{B} c^{3}}{2 \hbar G} A_{i} \Leftrightarrow S_{i}=\aleph_{i} \cdot \frac{k_{B}}{2}
$$

\subsection{On the Observed Flattening of Rotation Curves in Galaxies}

The concept of "Dark Matter" was proposed by Prof. F. Zwicky to explain the anomalous rotation curves of the galaxies. The problem was that, according to Newtonian dynamics, the velocities of any body of mass $m$ at a distance $r$ from the center of the galaxy, must be expressed by assuming a circular orbit, as: 


$$
\frac{m v^{2}}{r}-\frac{G M_{b} m}{r^{2}}=0 \Rightarrow v=\sqrt{\frac{G M_{b}}{r}}
$$

where $M_{b}$ is the baryonic mass of the Galaxy, $m$ the mass of the body, $G$ the gravitational constant, $r$ the distance of the body to the center of the galaxy and $V$ its tangential velocity.

However, the astronomical observations indicate that velocity of the rotation curves are flattened tending to a certain limit, instead of complying with the previous law where velocity must decrease as the radius $r$ increases.

Now, we consider an open system according to $\$ 5.3$, that is, any local system (i.e., Galaxy) within the Universe of $\aleph_{i}$ minimum masses or $N_{i}$ material particles of total mass: $M_{b}=N_{i} \cdot m=\aleph_{i} \cdot m_{\min }$, that form a closed surface of area $A$ at Time $t$. For simplicity, we will consider that the total mass $M_{b}$ is concentrated in the center of the surface. We will also consider that a material particle or body of mass $m$ is located on the surface at a distance $r$ from the center of the surface at Time $t$. Thus, $M_{b}$ is the baryonic mass of the Galaxy.

\subsubsection{Radius Upper Limit of a Gravitational System}

Now, we apply Mechanics to our local open system, this is: $\sum F_{i}=0$, according to Universe gravitational force Equation (70) which acts on every particle or body of mass $m$ causing a negative acceleration $a_{P}=-\frac{c}{t}$ and Equation (75) for gravitational force by assuming a circular orbit, we obtain at Time $t$ the equation:

$$
\frac{m v^{2}}{r}-\frac{G M_{b} m}{r^{2}}-\frac{G M m}{R^{2}}=0 \Rightarrow v^{2}=\frac{G M_{b}}{r}+\frac{c r}{t}
$$

However, this equation we can express it as follow and solving for $\left(\frac{G M_{b} t}{c r^{2}}\right)$, we obtain:

$$
\left.\begin{array}{l}
\text { 1) } v^{2}-\frac{2 c r}{t}=\frac{G M_{b}}{r}-\frac{c r}{t} \\
\text { 2) } \frac{c r}{v^{2} t}=\frac{1}{1+\frac{G M_{b} t}{c r^{2}}}
\end{array}\right\} \Rightarrow r^{2} \leq \frac{G M_{b} t}{c}
$$

which can be considered as the radius upper limit of a gravitational system at Time $t$, as for example a rotating Galaxy, that is:

$$
r^{2} \leq \frac{G M_{b} t}{c} \Rightarrow r_{\infty}^{2}=\frac{G M_{b} t}{c}
$$

${ }^{6} \mathrm{By}$ simplicity we denote $B=\frac{c r}{v^{2} t}$ and $y=\frac{G M_{b} t}{c r^{2}}$. Thus, we have by 2) that:

$B=\frac{1}{1+y} \Rightarrow B \leq 1, \forall y \geq 0$. By 1$)$ we have: $(1-2 B)=B(y-1) \Rightarrow(1-2 B) \leq(y-1) \Leftrightarrow B \leq 1$. Now, by 2) substituting value of $B$ in this equation and resolving for $y$, we obtain:

$$
(y-1) \leq\left(y^{2}-1\right) \Rightarrow\left(y^{2} \geq y\right) \Rightarrow(y \geq 1) \Rightarrow \frac{G M_{b} t}{c} \geq r^{2} .
$$




\subsubsection{Radius Lower Limit of a Gravitational System}

According to Equation (78) and Equation (79) we can obtain:

$$
v^{2}-\frac{2 G M_{b}}{r}=\frac{c r}{t}-\frac{G M_{b}}{r} \leq 0 \Rightarrow v^{2} \leq \frac{2 G M_{b}}{r}
$$

However, by Relativity any velocity $v \leq c$, that is, $v$ is always less or equal to the constancy of the speed of light in the vacuum. Thus, according to previous equation we obtain:

$$
\frac{1}{r} \leq \frac{c^{2}}{2 G M_{b}} \Leftrightarrow v \leq c \Rightarrow r \geq \frac{2 G M_{b}}{c^{2}}
$$

which establish a radius lower limit for a gravitational system at Time $t$ where we can verify that it corresponds to Schwarzschild radius.

\subsubsection{Velocity Lower Limit of a Gravitational System}

By Equation (79) for radius upper limit we obtain: $\frac{c r}{t} \leq \frac{G M_{b}}{r}$. Therefore according to Equation (78) and radius lower limit Equation (82) we can obtain:

$$
v^{2} \geq \frac{c r}{t} \Rightarrow v^{2} \geq \frac{2 G M_{b}}{c t}
$$

which represents the velocity lower limit for any gravitational system.

\subsubsection{Rotational Velocity Limit: Asymptotic Velocity}

According to Equation (78) we can express:

$$
\frac{c r^{2}}{t}=v^{2} r\left(1-\frac{G M_{b}}{v^{2} r}\right) \Rightarrow r=\frac{v^{2} t}{c}\left(1-\frac{G M_{b}}{v^{2} r}\right)
$$

Substituting this previous result for $r$ in Equation (81) we have:

$$
v^{4} \leq \frac{2 G M_{b} c}{t}\left(\frac{1}{1-\frac{G M_{b}}{v^{2} r}}\right)
$$

However, by Equation (81) we have:

$$
\left(\frac{1}{2} \leq \frac{G M_{b}}{v^{2} r}\right) \Rightarrow\left(\frac{1}{2} \leq 1-\frac{G M_{b}}{v^{2} r}\right) \Rightarrow\left(\frac{1}{1-\frac{G M_{b}}{v^{2} r}}\right) \leq 2 .
$$

Therefore we obtain the rotational velocity limit at Time $t$ as:

$$
v^{4} \leq \frac{4 G M_{b} c}{t} \Rightarrow v_{f}^{4}=\frac{4 G M_{b} c}{t}
$$

Therefore, we prove that rotational velocity limit or asymptotically flat rotation velocity $v_{f}$ depends on galaxy mass and Universe Time $t$ and not on its radius. Thus, the flatness velocity curves are due to the gravitational force of whole Universe Equation (70) acting on every material particle, which are gravitationally entangled. Therefore the missing mass is not missing.

$$
\text { Approaching } t_{0} \approx \frac{1}{H_{0}} \text { we obtain: } v^{4} \leq 4 G M_{b} c H_{0} \text {. For example: for }
$$


$H_{0}=(74.03 \pm 1.42) \mathrm{km} \cdot \mathrm{s}^{-1} \cdot \mathrm{Mpc}^{-1}$ in [7] and the baryonic mass of our Milky Way $M_{b} \simeq 1.3 \times 10^{41} \mathrm{~kg}$ in [11], the rotational velocity limit will be: $v<396 \mathrm{~km} / \mathrm{s}$, which is in agreement with the astronomical observations. We also indicate that dark matter could be, in principle, solved through the extended theories of gravity [6].

\subsection{On the Baryonic Tully-Fisher Relation}

The Tully-Fisher Relation was published by astronomers R. Brent Tully y J. Richard Fisher in 1977 and was originally posed as an empirical relation between optical luminosity and the width of the $21 \mathrm{~cm}$ line.

The Baryonic Tully-Fisher Relation (BTFR) is currently an empirical relation between baryonic mass and rotation velocity in disk galaxies [12], that is: $M_{b} \propto v^{4}$ and can be specifically expressed as:

$$
M_{b}=A v^{x}
$$

It's suffices to describe the data for rotating galaxies and where the parameters $A$ and $x$ should be adjusted according to astronomical observations being approximately for circular orbit: $x \cong 4$ and $A=\left(a_{0} G\right)^{-1}$ with $a_{0} \cong 1.2 \times 10^{-10} \mathrm{~m} \cdot \mathrm{s}^{-2}$.

Now, according to Equation (78) we can also express the rotational velocity $V$ at Time $t$ by two different ways as:

$$
\left[v^{2}=\frac{G M_{b}}{r}\left(1+\frac{c r^{2}}{G M_{b} t}\right) \vee v^{2}=\frac{c r}{t}\left(1+\frac{G M_{b} t}{c r^{2}}\right)\right]
$$

Therefore, by multiplying these two equations, the fourth power of the rotational velocity of the galaxy can be expressed at Universe Time $t$, as:

$$
v^{4}=a(r) G M_{b} \Leftrightarrow a(r)=\frac{c}{t}\left(1+\frac{c r^{2}}{G M_{b} t}\right)\left(1+\frac{G M_{b} t}{c r^{2}}\right)
$$

If we develop for $a(r)$ we obtain:

$$
a(r)=\frac{c}{t}\left(2+\frac{c r^{2}}{G M_{b} t}+\frac{G M_{b} t}{c r^{2}}\right)
$$

where we prove that $a(r)$ is a quadratic function. However, by Equation (85) or Equation (78) we could obtain that if: $r \rightarrow \infty \Rightarrow v \rightarrow \infty$, which is incompatible with Relativity since $v \leq c$ for any system in Nature. But according to Equation (80) for radius upper limit $r_{\infty}$ of a gravitational system at Time $t$, we obtain:

$$
a\left(r_{\infty}\right)=\frac{4 c}{t} \Rightarrow v_{f}^{4}=\frac{4 G M_{b} c}{t}
$$

which is according to Equation (83) and thus we obtain the asymptotically flat rotation velocity $v_{f}$ according to observations in typical galaxies.

$$
\text { It's remarkable by Equation (85) and } a\left(r_{\infty}\right)=\frac{4 c}{t} \text { that: }
$$




$$
v^{4} \in\left(\frac{2 G M_{b} c}{t}, \frac{4 G M_{b} c}{t}\right]
$$

Therefore, this suggests that Equation (84) remains valid for $x=4$ for $A \equiv$ constant .

However, the asymptotically flat rotation velocity $v_{f}$ Equation (83), can be expressed according to third equation of Equation (51) for Universe Time $t$ as a function of $H(t)=\frac{1}{t}\left(\frac{c^{2}}{v_{U}^{2}}-1\right)$ in the form:

$$
v_{f}^{4}=\frac{4 c H(t)}{\left(\frac{c^{2}}{v_{U}^{2}}-1\right)} G M_{b}
$$

Therefore, at current Universe Time $t_{0}$ where $H\left(t_{0}\right)=H_{0}$ and setting the expansion velocity of the universe $v_{U}$ as $1 / 5$ of the speed of light $c$, that is: $\frac{v_{U}}{c}=\frac{1}{5}$, then leads to the solution:

$$
v_{f}^{4}=a_{0} G M_{b} \Leftrightarrow a_{0}=\frac{c H_{0}}{6}
$$

which leads the Baryonic Tully-Fisher Relation (BTFR): $M_{b} \propto v^{4}$. For $H_{0}=(74.03 \pm 1.42) \mathrm{km} \cdot \mathrm{s}^{-1} \cdot \mathrm{Mpc}^{-1}$ in [7] one finds exactly $a_{0}=1.2 \times 10^{-10} \mathrm{~m} \cdot \mathrm{s}^{-2}$ which is the value predicted in different independent astronomical observations, e.g.: [12] [13] [14].

\subsection{Curvature of a Ray of Light by Gravity in the Vicinity of a Massive Object}

Let us now consider any relative reference system. We establish a light source located behind a object of mass $M$ at Time $t$ under the same conditions that section $\$ 5.3$. According to Equation (74), the work done by gravitational force $F=\left(-\frac{G M_{i} m}{r^{2}}\right)$ acting on any material particle $m$ during elapsed proper time interval $\Delta t$, causes an unrecoverable loss of energy. Thus, the action of gravity force $F$ affects the light's ray (photon mass) during a distance of the order of the mass's diameter $d=2 r=\Delta r$, where $r$ is the radius of the object at Time $t$ with $r>\frac{2 G M}{c^{2}}$ according to Equation (82) for Schwarzschild radius. Therefore, the light's ray loses energy and its trajectory cannot be a straight line.

When ray of light crosses said diameter, it deviates a vertical distance s corresponding to an angle $\alpha$. Therefore, the elapsed Time $\Delta t$ during distance $d=2 r$, will be: $\Delta t=\frac{2 r}{c}$. Thus, according to Equation (19) for the motion's equation we can express:

$$
s=s_{i}+v_{i} \Delta t+a(\Delta t)^{2} \Rightarrow s=a \frac{4 r^{2}}{c^{2}}
$$

where we consider $r_{i}=v_{i}=0$ as the particle's initial position and velocity at Time $t_{i}$ respectively. However, according to the principle of equivalence, Equa- 
tion (73): any gravitational acceleration is equivalent to an accelerated motion at Time $t$, that is, the complete physical equivalence of a gravitational acceleration and a corresponding acceleration of the reference system, then we can express now $a$ as a gravitational acceleration $a=\frac{G M}{r^{2}}$ at Time $t$ in previous equation and we obtain:

$$
s=\frac{4 G M}{c^{2}} \Rightarrow \alpha=\frac{4 G M}{r c^{2}}
$$

where $\alpha=\frac{s}{r}$ is the deflection angle at Time $t$, that is, the identical result predicted by General Relativity in [15].

\section{Curvature of a Ray of Light by Sun's Gravity}

If we take the radius and solar mass for example:

$$
\alpha=\frac{4 \cdot 6.674 \times 10^{-11} \mathrm{~N} \cdot \mathrm{kg}^{-2} \cdot \mathrm{m}^{2} \times 1.99 \times 10^{30} \mathrm{~kg}}{6.957 \times 10^{8} \mathrm{~m} \times\left(3 \times 10^{8} \mathrm{~m}\right)^{2}}=8.4847 \times 10^{-6} \text { radians }
$$

We know that radian $=\frac{360^{\circ}}{2 \pi}$ and $1^{\circ}=3600^{\prime \prime}$ (seconds), thus:

$$
\alpha=8.4847 \times 10^{-6} \cdot \frac{360^{\circ}}{2 \pi}=\left(4.8614 \times 10^{-4}\right)^{\circ}=1.75^{\prime \prime}
$$

We have obtained the value according to astronomical observations (Eddington 1920), that is, a light ray grazing the surface of the Sun is deflected by 1.75 arc seconds.

\section{Quantum and Statistical Part}

\subsection{Statistical Interpretation of Entropy-Action Equivalence Law}

We consider that with regard to any relative reference system, a particle of mass $m$ experiences during any interval of proper time $\Delta t=t-t_{i}$ con $\left(t>t_{i}\right)$ by Equation (10), an increase of entropy $\Delta S=S(E, t)-S\left(E_{i}, t_{i}\right)$ according Equation (61) that we can express as:

$$
S(E, t)=S\left(E_{i}, t_{i}\right)+k_{B} \frac{m c^{2}}{\hbar} \Delta t \Rightarrow S(E, t)=k_{B}\left(\frac{S\left(E_{i}, t_{i}\right)}{k_{B}}+\frac{m c^{2}}{\hbar} \Delta t\right)
$$

where $S(E, t), m y \quad E=m c^{2}$ is the entropy, mass and energy of the particle at Time $t$, and $S\left(E_{i}, t_{i}\right), m_{i}$ y $E_{i}=m_{i} c^{2}$ are the entropy, mass and energy of the particle at Time $t_{i}$, because as we proved, any material particle must be lost, with regard to any relative reference system, at every interval of proper time $\Delta t$ an irrecoverable part of mass/energy as Time progresses.

Obviously the entropy $S\left(E_{i}, t_{i}\right)$ is defined during the interval of proper time: $\Delta t_{i j}=t_{i}-t_{j}$ with $\left(t_{i}>t_{j}\right)$ as:

$$
S\left(E_{i}, t_{i}\right)=S\left(E_{j}, t_{j}\right)+k_{B} \frac{m_{i} c^{2}}{\hbar} \Delta t_{i j}
$$


and so on. However, this Equation (86) we can express it as:

$$
S(E, t)=k_{B} \cdot \ln \left[W\left(E_{i}, t_{i}\right) \cdot W(E, \Delta t)\right] \Rightarrow S(E, t)=k_{B} \cdot \ln [W(E, t)]
$$

according to Boltzmann's statistical entropy [16] and where obviously

$$
\begin{aligned}
& W(E, t)=\mathrm{e}^{\frac{S(E, t)}{k_{B}}} \text {. Thus: } \\
& W(E, t)=W\left(E_{i}, t_{i}\right) \cdot \mathrm{e}^{\frac{m c^{2}}{\hbar} \Delta t} \Leftrightarrow\left(W\left(E_{i}, t_{i}\right)=\mathrm{e}^{\frac{S\left(E_{i}, t_{i}\right)}{k_{B}}} \wedge W(E, \Delta t)=\mathrm{e}^{\frac{m c^{2} \cdot \Delta t}{\hbar}}\right)
\end{aligned}
$$

and therefore we can conclude that:

- $W(E, t)$ is the total number of possible states for the material particle $m$ at Universe's Time $t$.

- $W\left(E_{i}, t_{i}\right)$ is the total number of possible states for the material particle $m_{i}$ at Universe's Time $t_{i}$.

- $W(E, \Delta t)$ is the total volume of configurations or possible states of the material particle $m$ during the elapsed interval of proper time $\Delta t=t-t_{i}$.

\section{Probability}

We consider now the total volume of possible states for any material particle $m$ at Universe Time $t$, that is: $W(E, t)$. Let us ask now: What is the probability of finding to the material particle at Time $t$ in a unique state within the total volume of possible configurations where every state has the same probability? In this case, the situation is similar to having at Time $t$ a closed box full of " $n$ " balls every one of them labeled as " 1 " to " $n$ " and find the probability of extracting at Time $t$ any number, for example number "14". Obviously probability is: $p=1 / n$.

Therefore, according Equation (87) we can express that probability of finding to the particle $m$ at Universe Time $t$ at any given state of energy as:

$$
P(E, t)=\frac{1}{W(E, t)} \Rightarrow P(E, t)=P\left(E_{i}, t_{i}\right) \cdot \mathrm{e}^{-\frac{E \cdot \Delta t}{\hbar}}
$$

where probability $P\left(E_{i}, t_{i}\right)$ of finding to particle $m_{i}$ at Universe Time $t_{i}$ at any given state will be:

$$
P\left(E_{i}, t_{i}\right)=\frac{1}{W\left(E_{i}, t_{i}\right)} \Rightarrow P\left(E_{i}, t_{i}\right)=P\left(E_{j}, t_{j}\right) \cdot \mathrm{e}^{-\frac{E_{i} \cdot \Delta t_{i j}}{\hbar}}
$$

where obviously the interval of proper time is: $\Delta t_{i j}=t_{i}-t_{j}$ with $\left(t_{i}>t_{j}\right)$ and $P\left(E_{j}, t_{j}\right)$ the probability of finding to particle $m_{j}$ at any given state at Universe Time $t_{j}$ and so on. Thus, the probability at any proper time interval $\Delta t=t-t_{i}$ depends of the probability on the previous proper time interval $\Delta t_{i j}=t_{i}-t_{j}$.

\subsection{Discrete Wave Function}

According to previous section, we consider a material particle $m$ with regard to any relative reference system. The following reflections will be made according to the Wave Mechanics. By Equation (26) we can write: 


$$
E=m c^{2}=h v \Rightarrow E=\hbar w \quad \Leftrightarrow \quad w=2 \pi v
$$

where $w$ is the angular velocity at Time $t$. However, by Equation (27) we can also express the energy of the particle at Time $t$ as:

$$
E=m c^{2}=m \boldsymbol{v} \cdot \boldsymbol{v}_{p} \Leftrightarrow \boldsymbol{v} \cdot \boldsymbol{v}_{p}=c^{2}
$$

We now consider $\boldsymbol{p}=m \boldsymbol{v}=\hbar \boldsymbol{k}$ by Equation (27), where $\boldsymbol{k}=2 \pi / \lambda$ is the wave vector at Universe Time $t$. If we denote $\Delta \boldsymbol{r}=\boldsymbol{v}_{p} \cdot \Delta t$ as displacement of the wave during interval of proper time $\Delta t$, then the action $E \cdot \Delta t$ can be expressed as:

$$
m c^{2} \cdot \Delta t=\hbar \boldsymbol{k} \cdot \Delta \boldsymbol{r}
$$

Therefore, we can now also express the probability of finding to the particle $m$ at Universe Time $t$ at any given state of energy or position as:

$$
P(E, t)=P\left(E_{i}, t_{i}\right) \cdot \mathrm{e}^{-\frac{E \cdot \Delta t}{\hbar}} \Leftrightarrow P(\boldsymbol{r}, t)=P\left(\boldsymbol{r}_{i}, t_{i}\right) \cdot \mathrm{e}^{-\boldsymbol{k} \Delta \boldsymbol{r}}
$$

Thus, probability of finding to the particle $m$ at any given state at Universe Time $t$ combining Equation (88) and Equation (89), can be expressed now as:

$$
P(\boldsymbol{r}, t)=P\left(\boldsymbol{r}_{j}, t_{j}\right) \cdot \mathrm{e}^{\left(-\boldsymbol{k}_{i} \Delta \boldsymbol{r}_{i}-w \Delta t\right)}
$$

where $\boldsymbol{k}_{i}$ is the wave vector ${ }^{7}$ at Time $t_{i}, \Delta \boldsymbol{r}_{i}=\boldsymbol{v}_{p i} \cdot \Delta t_{i j}$ are the displacement and velocity $\boldsymbol{v}_{p i}$ of the wave during interval of proper time $\Delta t_{i j}=t_{i}-t_{j}$. Obviously, the probability at Time $t$ during the elapsed proper time interval $\Delta t=t-t_{i}$ depends of the probability at Time $t_{i}$ during the previous elapsed proper time interval $\Delta t_{i j}=t_{i}-t_{j}$.

However, we can raise this previous equation to the power of imaginary number $i$ which we denote as $\Psi(\boldsymbol{r}, t)=P(\boldsymbol{r}, t)^{i}$ as a complex function at Universe Time $t$, then the previous equation can be expressed as:

$$
\Psi(\boldsymbol{r}, t)=\Psi\left(\boldsymbol{r}_{j}, t_{j}\right) \cdot \mathrm{e}^{i\left(-\boldsymbol{k}_{i} \Delta \boldsymbol{r}_{i}-w \Delta t\right)}
$$

where $\Psi\left(\boldsymbol{r}_{j}, t_{j}\right)=P\left(\boldsymbol{r}_{j}, t_{j}\right)^{i}$ and we verify it has the form of a discrete complex wave function.

It's remarkable that function value $\Psi(\boldsymbol{r}, t)$ at Time $t$ during the elapsed proper time interval $\Delta t=t-t_{i}$ depends on the function value at Time $t_{i}$ during the previous elapsed proper time interval $\Delta t_{i j}=t_{i}-t_{j}$, that is: $\Psi\left(\boldsymbol{r}_{i}, t_{i}\right)=\Psi\left(\boldsymbol{r}_{j}, t_{j}\right) \cdot \mathrm{e}^{i\left(-\boldsymbol{k}_{i} \Delta \boldsymbol{r}_{i}\right)}$.

\subsubsection{Schrödinger Equation as an Approximation to Continuum} We can approximate Equation (90) to a continuum form according to:

- The interval of proper time is continuum according to the current physics, that is, considering the fundamental minimum time $\tau=0$ and therefore $\Delta t \simeq \Delta t_{i j}$.

- The energy of the particle does not change in Time, that is, does not lose

${ }^{7}$ Since the wave vector is a vector pointing in the direction of propagation of the wave, we could have considered that the motion is in the positive direction and therefore $\boldsymbol{k}_{i} \Delta \boldsymbol{r}_{i}$ would have been positive. 
energy as time progresses and therefore remains constant without any force acting on it as time progresses.

Since obviously $\Psi\left(\boldsymbol{r}_{j}, t_{j}\right) \equiv$ cte during the interval of proper time $\Delta t=t-t_{i}$, then we can express $\Psi(\boldsymbol{r}, t)$ as:

$$
\Psi(\boldsymbol{r}, t)=\Psi_{0} \cdot \mathrm{e}^{i(-\boldsymbol{k} \cdot \boldsymbol{r}-w t)}
$$

with $\Psi_{0}=\Psi\left(\boldsymbol{r}_{j}, t_{j}\right)$ and where now $t$ is any continuum interval of proper time. Thus, we verify that this equation is the De Broglie wave function, which we can derive:

$$
\frac{\partial}{\partial t} \Psi(\boldsymbol{r}, t)=-i w \Psi(\boldsymbol{r}, t), \quad \nabla \Psi(\boldsymbol{r}, t)=-i k \Psi(\boldsymbol{r}, t), \quad \nabla^{2} \Psi(\boldsymbol{r}, t)=-k^{2} \Psi(\boldsymbol{r}, t)
$$

and therefore we obtain the famous Schrödinger Equation.

$$
-\frac{\hbar^{2}}{2 m} \nabla^{2} \Psi(\boldsymbol{r}, t)+V(\boldsymbol{r}, t) \Psi(\boldsymbol{r}, t)=i \hbar \frac{\partial}{\partial t} \Psi(\boldsymbol{r}, t)
$$

where $E=\frac{p^{2}}{2 m}+V(\boldsymbol{r}, t)$ is the classical approximation as non-relativistic energy.

\subsubsection{Probability Wave Function}

Both Equation (90) for the discrete wave function and Equation (91) for continuum wave function is established that $\Psi(\boldsymbol{r}, t)=P(\boldsymbol{r}, t)^{i}$. However, we can denote $z=\ln (\Psi(\boldsymbol{r}, t))=i \cdot \ln (P(\boldsymbol{r}, t))$. Therefore we can obtain:

$$
|z|=|\ln (\Psi(\boldsymbol{r}, t))|=\sqrt{0+[\ln (P(\boldsymbol{r}, t))]^{2}} \Rightarrow|\ln (\Psi(\boldsymbol{r}, t))|^{2}=[\ln (P(\boldsymbol{r}, t))]^{2}
$$

But we know that:

$$
|\ln (\Psi(\boldsymbol{r}, t))|^{2}=(\ln |\Psi(\boldsymbol{r}, t)|)^{2} \Rightarrow(\ln |\Psi(\boldsymbol{r}, t)|)^{2}=[\ln (P(\boldsymbol{r}, t))]^{2}
$$

And therefore we obtain:

$$
|\Psi(\boldsymbol{r}, t)|=P(\boldsymbol{r}, t)
$$

That is, the wave function module at Time $t$ is equal to probability in the same Time $t$, according to Born's probabilistic interpretation.

\section{Acknowledgements}

This paper is dedicated to my sister Rocío.

\section{Conflicts of Interest}

The author declares no conflicts of interest regarding the publication of this paper.

\section{References}

[1] Yang, Y., Peng, C., Zhu, D., Buljan, H., Joannopoulos, J.D., Zhen, B. and Soljacic, M. (2019) Synthesis and Observation of Non-Abelian Gauge Fields in Real Space. Science, 365, 1021-1025. https://doi.org/10.1126/science.aay3183 
[2] Faizal, M., Khalil, M.M. and Das, S. (2016) Time Crystals from Minimum Time Uncertainty. The European Physical Journal C, 76, 30. https://doi.org/10.1140/epjc/s10052-016-3884-4

[3] Weinberg, S. (1972) Gravitation and Cosmology: Principles and Applications of the General Theory of Relativity. Wiley, New York.

[4] Ballantine, K.E., Donegan, J.F. and Eastham, P.R. (2016) There Are Many Ways to Spin a Photon: Half-Quantization of a Total Optical Angular Momentum. American Association for the Advancement of Science: Science Advances, 2, e1501748. https://doi.org/10.1126/sciadv.1501748

[5] Hooft, G. (1993) Dimensional Reduction in Quantum Gravity.

[6] Corda, C. (2009) Interferometric Detection of Gravitational Waves: The Definitive Test for General Relativity Christian Corda. International Journal of Modern Physics D, 18, 2275-2282. https://doi.org/10.1142/S0218271809015904

[7] Riess, A.G., Casertano, S., Yuan, W.L., Macri, L.M. and Scolnic, D. (2019) Large Magellanic Cloud Cepheid Standards Provide a 1\% Foundation for the Determination of the Hubble Constant and Stronger Evidence for Physics beyond $\Lambda \mathrm{CDM}$. The Astrophysical Journal, 876, 85.

https://doi.org/10.3847/1538-4357/ab1422

[8] Bekenstein, J.D. (1981) Universal Upper Bound on the Entropy-to-Energy Ratio for Bounded Systems. Physical Review D, 23, 287.

https://doi.org/10.1103/PhysRevD.23.287

[9] Verlinde, E. (2011) On the Origin of Gravity and the Laws of Newton. JHEP, 1104, 029. https://doi.org/10.1007/JHEP04(2011)029

[10] Mureika, J.R. and Mann, R.B. (2010) Does Entropic Gravity Bound the Masses of the Photon and Graviton?

[11] Boylan-Kolchin, M., et al. (2013) The Space Motion of Leo I: The Mass of the Milky Way's Dark Matter Halo. The Astrophysical Journal, 768, Article ID: 140. https://doi.org/10.1088/0004-637X/768/2/140

[12] McGaugh, S.S. (2011) The Baryonic Tully-Fischer Relation of Gas Rich Galaxies as a Test of $\Lambda$ CDM and MOND. Astronomical Journal, 143, 40.

[13] McGaugh, S.S., Lelli, F. and Schombert, J. (2017) The Radial Acceleration Relation in Rotationally Supported Galaxies. Physical Review D, 96, Article ID: 083523. https://doi.org/10.1103/PhysRevLett.117.201101

[14] Smolin, L. (2017) MOND as a Regime of Quantum Gravity. Physical Review D, 96, Article ID: 083523.

[15] Bartelmann, M. and Schneider, P. (2001) Weak Gravitational Lensing. Physics Reports, 340, 291-472. https://doi.org/10.1016/S0370-1573(00)00082-X

[16] Gujrati, P.D. (2015) On Equivalence of Non-Equilibrium Thermodynamic and Statistical Entropies. Entropy, 17, 721. https://doi.org/10.3390/e17020710 